\title{
A moving-mirror gonioscope for retinal surgery
}

\author{
DAVID OWEN \\ London
}

SUMMARY A moving-mirror gonioscope for use in retinal surgery is described, and its advantages are discussed.

The advantages of the use of the operating microscope in surgery of the anterior segment of the eye are well known. To attempt to gain these advantages in surgery of the retina and vitreous must involve the use of a contact lens to neutralise the curvature of the cornea. In addition provision must be made to allow examination of the periphery of the fundus. This paper describes a new instrument designed to fulfil these purposes.

\section{Description}

The contact lens consists of a plano-concave lens, diameter $12 \mathrm{~mm}$, radius of curvature $7.5 \mathrm{~mm}$, mounted in the base of the instrument. The walls of the instrument, composed of black Perspex, enclose an air-filled space, in which is mounted the single movable mirror. The mirror is rectangular in shape,

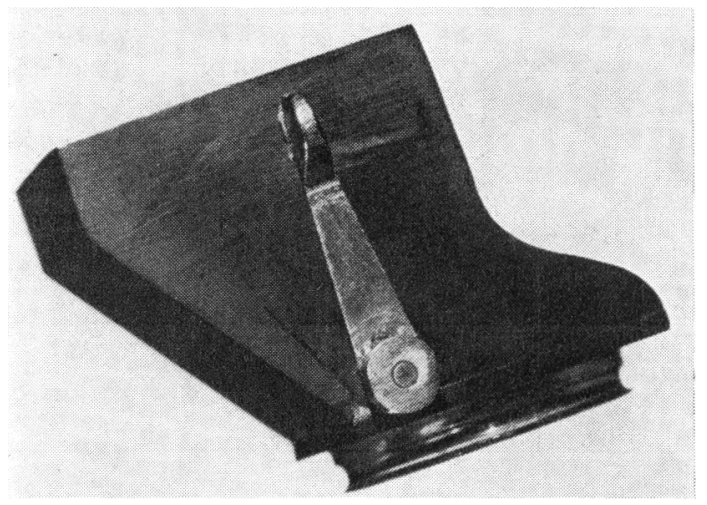

and is attached at its lower edge to a transverse spindle. This spindle can be rotated by moving the arm on the exterior of the instrument, and in this way the angle of the mirror can be altered at will. The overall shape of the instrument reflects the fact

Address for reprints: Mr D. A. Owen, Ashford Hospital, Ashford, Middlesex

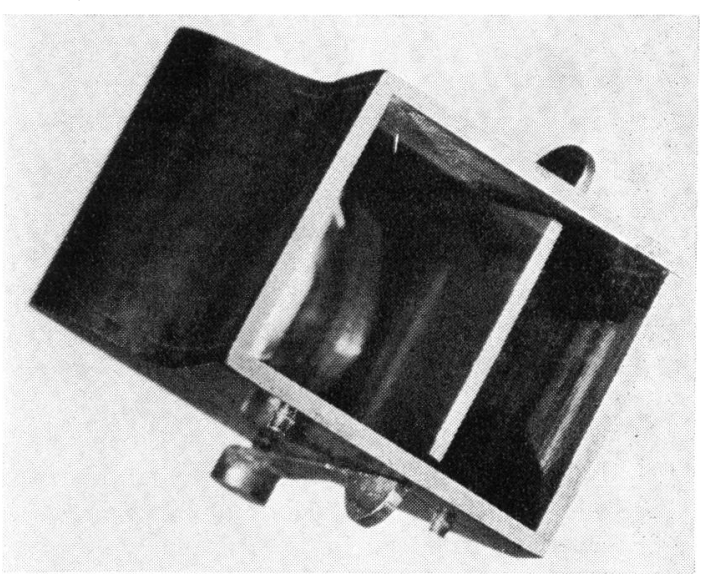

that only one mirror is used, and is designed to minimise obstruction to the surgeon. The roof of the instrument is formed by a thin rectangular sheet of Perspex, but this functions merely as a dust-cover, for it is removed when the instrument is in use.

\section{Use}

The instrument is designed to be used in theatre on the anaesthetised patient. The pupil must be fully dilated. Lid retractors or sutures are used in the usual way, and the instrument is placed on the cornea. To avoid air bubbles a jet of saline from a syringe is directed just beneath the instrument as it is lowered on to the cornea, a manoeuvre which is not difficult to accomplish. The surgeon continues to hold the instrument in contact with the cornea, the most convenient grip being with the thumb and middle finger, thus allowing the index finger freedom to adjust the angle of the mirror by pushing the lever on the exterior of the instrument backwards or forwards as desired. This procedure takes a little practice.

The surgeon then adjusts the microscope to obtain a view of the fundus. A slit beam is required, 
with its axis just a few degrees off the viewing axis, and the surgeon finds the optimum arrangement in exactly the same way as in the case of the Goldman three-mirror lens. The motorised traversible slit illuminator of Carl Zeiss, Oberkochen, is excellent for this purpose, and an X-Y coupling mechanism is also most helpful.

The surgeon's free hand may be used to indent the sclera, for which purpose finely-toothed forceps are convenient. It is sometimes necessary to alter the position of the instrument relative to the eyeball a millimetre or two, and, in practice, it is found easier to keep the instrument still and rotate the eyeball beneath it by means of toothed forceps gripping the episcleral tissues (the same forceps being used for indentation).

The periphery of the fundus is examined by directing the slit beam on to the mirror of the instrument, and a more anterior or a more posterior view in that particular meridian is obtained by adjusting the angle of the mirror. With indentation the ora serrata and pars plana may readily be seen, and with the mirror as far back as it will go the angle of the anterior chamber may be examined. Different meridians are examined merely by rotating the instrument on the globe. The sense of continuity thus achieved is very helpful to the surgeon in orientation and in the interpretation of what he sees. The optic disc and posterior pole can be examined by directing the beam straight through the contact lens, i.e., in front of the lower edge of the mirror.

\section{Discussion}

The advantages of contact lens biomicroscopy over other methods of examination of the fundus have been described elsewhere (Goldman, 1949; Schirmer, 1970). The chief advantages are the greater magnification afforded, the greater information a strong slit beam provides, especially in respect of threedimensional appreciation, and a reduction of the effect of scatter from opacities of the media as compared with coaxial illumination. The use of an instrument with a single adjustable mirror avoids the loss of continuity which results from the use of an instrument with multiple fixed mirrors. Frisen (1972) has described an ingenious instrument incorporating one fixed and one adjustable mirror. The instrument described here, however, has been designed specifically for use in the operating theatre in the hope that it will enable the operating microscope to be more effectively used in retinal surgery.

I am much indebted to Mr G. Nissel and company, and to $\mathrm{Mr}$ M. Ruben and the contact lens department, Moorfields, for constructing the prototypes. I am also most grateful to Mr A. G. Bennett, of City University, for much helpful advice on the optics of the instrument.

\section{References}

Frisen, L. (1972). Archives of Ophthalmology, 87, 202.

Goldman, H. (1949). British Journal of Ophthalmology, 33. 242.

Schirmer, K. (1970). Archives of Ophthalmology, 84, 66. 